\title{
e Long-term Postpartum Headache: PDPH Associated with Major Depression
}

\section{TO THE EDITOR}

Both headache and major depression are relatively common during the postpartum period and can have devastating consequences on the mother and child. If postpartum headache is a symptom of major depression, it may also increase emotional distress and depressive symptoms. We present a clinical case of a woman with long-term postpartum headache associated with major depression but also postdural puncture headache (PDPH).

A 35-year-old Caucasian woman, gravida 2 para 2, was admitted with her son to our Mother and Baby Unit for a major depressive episode after delivery. She had no history of somatic disorders, family or personal psychiatric history, or substance abuse. One month before giving birth, pre-eclampsia developed and an emergency cesarean delivery was performed. Spinal anesthesia was performed at the L3-L4 interspace with a Vygon $27 \mathrm{G}$ needle. She gave birth to a little boy. Nicardipine and labetalol were started. One week after delivery, the patient complained of headache and hemifacial paresthesia. Blood serum screening (including thyroid hormones, glucose and calcium plasma concentration) and cerebral contrast magnetic resonance imaging (MRI) eliminated intracranial venous thrombosis, hemorrhage, and meningitis. The working diagnosis was tension-type headache potentiated by hypertension. However, whereas blood pressure improved, headache worsened. She described major asthenia, poor energy and concentration levels, and major anxiety. Thus, major depression was diagnosed. She was hospitalized with her son in our Mother and Baby Unit. Her treatment was escitalopram $15 \mathrm{mg} / \mathrm{j}$, atenolol 100 $\mathrm{mg} / \mathrm{d}$, and losartan $100 \mathrm{mg} / \mathrm{d}$.

She presented with major anxiety, asthenia, difficulty in falling asleep, anhedonia, and sadness. She reported having difficulties bonding with her son and feeling guilty about that. She had no suicidal or infanticidal ideation. Standard rating scales to assess the severity of depression showed a severe level of symptoms: the score of the 17-item Hamilton Depression Rating Scale (HAMD-17) was 27, and the score of the Edinburgh Postnatal Depression Scale (EPDS) was 19. Interestingly, several items of the HAMD-17 indicated somatic symptoms.
She complained of frontal and occipital headache of the right side irradiating to the neck and shoulders. Her headache increased with sitting or standing up, and abated when lying down. Headache was accompanied by a stiff neck, hyperacusis, and visual impairment. She described an episode of tinnitus. Arterial blood pressure was normal. No evidence of focal disorder was present. PDPH was suspected. MRI showed both cerebellar tonsils moving down. Despite administration of pregabalin $50 \mathrm{mg} / \mathrm{d}$, thiocolchicoside $8 \mathrm{mg} / \mathrm{d}$ combined with paracetamol and tramadol chlorhydrate on demand, no relief of headache occurred. An epidural blood patch (EBP) was performed with $30 \mathrm{~mL}$ of autologous blood and led to a dramatic improvement of the headache. However, sadness, anxiety, anhedonia, and sense of guilt remained. After EBP, HAMD-17 was 22.

Clomipramine was introduced and was progressively increased to $100 \mathrm{mg} /$ day. All other treatments were progressively decreased and discontinued 4 weeks later.

Mother-child interactions were good. The mother was able to meet his primary needs. The baby presented with a good psychomotor development. The HAMD-17 score was 5, and EPDS was 3 at discharge. No depressive relapse had occurred after 6 months.

This case report illustrates the importance of searching for a somatic disease cause to explain symptoms, even in presence of major depression, and treating it parallel to the psychiatric care. A somatic disease may be risk factor for depression (1) and headache could be a physical symptom of post-partum depression. Postpartum depression (estimated incidence: 14.5\%) is associated with negative physical and mental health outcomes for both the mother and infant (2).

Tension headache due to depression was evoked in our patient. Lack of improvement of symptoms despite numerous analgesics and increased incidence of headache after a difficult delivery could evoke somatization disorders. Most depressed patients focus their distress on somatic symptoms and more than half report unexplained physical symptoms. The major impact of headache, namely preventing the mother from taking care of her baby, could move towards an organic cause.

Headache is relatively common following child- 
birth. More than one third of women report the occurrence of headache during the first week after delivery. Primary headaches are the commonest causes. However, causes of secondary headache (pre-eclampsia, cerebral vein thrombosis, PDPH, reversible cerebral vasoconstriction syndrome, intracranial tumor, pituitary apoplexy, hemorrhage, ischemic stroke, meningitis) should be promptly diagnosed and treated (3). PDPH is the most common complication of obstetric regional anesthesia and the most effective treatment is EBP. A meta-analysis reported a risk of $1.5 \%$ accidental dural puncture after epidural insertion, and $52 \%$ of these will result in PDPH (4). Whereas patients with anxiety and depression had similar PDPH rates to healthy controls (5), a past history of depression was associated with the increased duration of PDPH (6).

The relationship between headache and depression seems a bidirectional one. Depression can be both a cause and a result of pain. Mothers reporting persistent perinatal pain (vagina, caesarean incision site, breasts, back, severe headaches) are at increased risk of developing post-partum depression (7). Interestingly, in major depressive disorders, the severity of depression, headache intensity, and incidence have been correlated with each other (8). Additionally, about half of patients report headache worsening during or after a depressive episode (8). In a large cross-sectional population-based study including 47,257 patients (9), depression was associated with migraine and non-migrainous headache when compared with headache-free individuals. Patients with major depressive disorders and comorbid migraine were reported to have higher physical and anxiety scores than patients without migraine. Moreover, in patients with anxiety and mood disorders, a higher severity of the psychopathologic disease has been associated with greater perception of pain severity (10). Women who do not receive optimal pain management are at increased risk for depression (7).

Women with postpartum depression should be evaluated for acute and chronic perinatal pain. Indeed, pain and depression can limit the interactions between the mother and her baby. Physical, functional, and emotional health status should be assessed in women following childbirth and all components should be treated.

Antonia Mezzacappa, MD

Department of Psychiatry

Assistance Publique-Hôpitaux de Paris

Bicêtre University Hospital
78 rue du Général Leclerc

94275 Le Kremlin Bicêtre, France

Isabelle Nègre, MD

Pain center

Assistance Publique-Hôpitaux de Paris

Bicêtre University Hospital

78, rue du Général-Leclerc

94275 Le Kremlin-Bicêtre cedex, France

Cécile Jean-Baptiste

Department of Psychiatry

Assistance Publique-Hôpitaux de Paris

Bicêtre University Hospital

78 rue du Général Leclerc

94275 Le Kremlin Bicêtre, France

Odile Cazas, MD

Department of Psychiatry

Assistance Publique-Hôpitaux de Paris

Bicêtre University Hospital

78 rue du Général Leclerc

94275 Le Kremlin Bicêtre, France

Patrick Hardy, MD, PhD

Department of Psychiatry

Assistance Publique-Hôpitaux de Paris

Bicêtre University Hospital

78 rue du Général Leclerc

94275 Le Kremlin Bicêtre, France

Inserm UMR 1178

Univ Paris Sud

78 rue du Général Leclerc

94275 Le Kremlin Bicêtre, France

Dan Benhamou, MD, PhD

Dept. of Anaesthesia and Intensive Care Medicine

Assistance Publique-Hôpitaux de Paris

Bicêtre University Hospital

78, rue du Général-Leclerc

94275 Le Kremlin-Bicêtre cedex, France

Inserm UMR 1195,

Petites molécules de neuroprotection

neurorégénération et remyélinisation

Univ Paris-Sud,

78 rue du Général Leclerc

94275 Le Kremlin Bicêtre, France 
Florence Gressier, MD, PhD

Department of Psychiatry

Assistance Publique-Hôpitaux de Paris

Bicêtre University Hospital

78 rue du Général Leclerc

94275 Le Kremlin Bicêtre, France
Inserm UMR 1178

Univ Paris Sud

78 rue du Général Leclerc

94275 Le Kremlin Bicêtre, France

E-mail address: florence.gressier@aphp.fr

\section{References}

1. Webb DA, Bloch RJ, Coyne JC, Chung EK, Bennett IM, Flatow Culhane J. Postpartum physical symptoms in new mothers: Their relationship to functional limitations and emotional well-being. Birth 2008; 35:179-187.

2. Weinberg MK, Tronick EZ. The impact of maternal psychiatric illness on infant development. J Clin Psychiatry 1998; 59:5361.

3. Lim SY, Evangelou N, Jürgens S. Postpartum headache: Diagnostic considerations. Pract Neurol 2014; 14:92-99.

4. Choi PT, Galinski SE, Takeuchi L, Lucas S, Tamayo C, Jadad AR. PDPH is a common complication of neuraxial blockade in parturients: A meta-analysis of obstetrical studies. Can J Anesth 2003; 50:460-
469.

5. Dodge HS, Ekhator NN, Jefferson-Wilson L, Fischer M, Jansen I, Horn PS, Hurford WE, Geracioti TD. Cigarette smokers have reduced risk for post-dural puncture headache. Pain Physician 2013; 16:E25-E30.

6. Van Oosterhout WP, van der Plas AA, van Zwet EW, Zielman R, Ferrari MD, Terwindt GM. Postdural puncture headache in migraineurs and nonheadache subjects: A prospective study. Neurology 2013; 80:941-948.

7. Gaudet C, Wen SW, Walker MC. Chronic perinatal pain as a risk factor for postpartum depression symptoms in Canadian women. Can J Public Health. 2013; 104:e375-e387.
8. Hung Cl, Liu CY, Wang SJ. Precipitating or aggravating factors for headache in patients with major depressive disorder. J Psychosom Res 2008; 64:231-235.

9. Zwart JA, Dyb G, Hagen K, Ødegård KJ, Dahl AA, Bovim G, Stovner LJ. Depression and anxiety disorders associated with headache frequency. The NordTrøndelag Health Study. Eur J Neurol 2003; 10:147-152.

10. Pompili M, Innamorati M, Serafini G, Gonda X, Campi S, Rapinesi C, Giordano G, Montebovi F, Palermo M, Giupponi G, Tatarelli R, Biondi M, Amore M, Sher L, Rihmer Z, Girardi P. How does subjective experience of pain relate to psychopathology among psychiatric patients? Gen Hosp Psychiatry 2012; 34:534-540. 